\title{
Herbal Medicine, Hachimi-jio-gan, and Its Component Cinnamomi Cortex Activate the Peroxisome Proliferator- activated Receptor Alpha in Renal Cells
}

\author{
TSUYOSHI MONDEN, TAKESHI HOSOYA*, YASUYO NAKAJIMA*, MIKIKO KISHI*, TETUROU SATOH*, \\ Koshi HASHIMOTO*, KIKUO KASAI, MASANOBU YAMADA* AND MASATOMO MORI* \\ Department of Endocrinology and Metabolism, Dokkyo University School of Medicine, Mibu, Tochigi 321-0293 Japan \\ *Department of Medicine and Molecular Science, Gunma University Graduate School of Medicine, Maebashi, Gunma $371-8511$ \\ Japan
}

\begin{abstract}
Hachimi-jio-gan is widely used to improve several disorders associated with diabetes, but its mechanism remains poorly understood. In an attempt to clarify the mechanism of Hachimi-jio-gan, we investigated the effects of this herbal medicine and its components in transfection studies of CV1 cells, especially nuclear receptor-mediated actions. One half $(0.5) \mathrm{mg} / \mathrm{ml}$ of Hachimi-jio-gan activated peroxisome proliferator-activated receptor (PPAR $\alpha$ ), mediating the activation by 3.1-fold on DR1 response elements; however, it did not affect PPAR $\gamma$, thyroid hormone receptor, androgen receptor, estrogen receptor or RXR. In addition, this activation was observed in a dose-dependent manner. Next, to determine which components of Hachimi-jio-gan activate PPAR $\alpha$-mediated transcription, 8 of its components (rehmanniae radix, orni fructus, dioscoreae rhizoma, alismatis rhizoma, hoelen, moutan cortex, cinnamomi cortex, aconiti) were tested. Only cinnamomi cortex $(1.0 \mathrm{mg} / \mathrm{ml})$ increased PPAR $\alpha$-mediated transcription by 4.1 -fold, and this activation was specific for PPAR $\alpha$, and not for other nuclear receptors. Moreover, this PPAR $\alpha$-related activation by cinnamomi cortex is specifically observed in renal cells. Taken together, these findings indicate that Hachimi-jio-gan and cinnamomi cortex may have a pharmacological effect through the target site for PPAR $\alpha$.
\end{abstract}

Key words: Hachimi-jio-gan, cinnamomi cortex, PPAR $\alpha$

(Endocrine Journal 55: 529-533, 2008)

IN Japan and China, traditional herbal medicines (Kampo) have often been used for diabetes mellitus to treat symptoms such as fever, numbness, and edema. In particular, Hachimi-jio-gan is used clinically to improve diabetic nephropathy [1-4]. Recently, Hachimijio-gan was reported to suppress hyperglycemia via insulin production and secretion from the pancreas [5]. In addition, Hachimi-jio-gan has been widely used for the treatment of many chronic diseases such as renal nephritis [6], vegetative ataxia [7], major depressive

Received: October 17, 2007

Accepted: October 23, 2007

Correspondence to: Tsuyoshi MONDEN, M.D., Ph.D., Department of Endocrinology and Metabolism, Dokkyo University School of Medicine, 880 Kitakobayashi, Mibu, Shimotsuga-gun, Tochigi 321-0293, Japan disorder [8, 9], hyperprolactinemia [10], and hyperlipidemia [11]; however, the mechanisms of Hachimijio-gan remain unclear.

The objective of this study was to investigate whether Hachimi-jio-gan has functions related to hormonal effects using transfection assays with thyroid hormone receptor, androgen receptor, estrogen receptor, estrogen receptor, RXR, PPAR $\gamma$, and PPAR $\alpha$ into several cell lines. Moreover, Hachimi-jio-gan is composed of eight ingredients, rehmanniae radix, orni fructus, dioscoreae rhizoma, alismatis rhizoma, hoelen, moutan cortex, cinnamomi cortex and aconiti; therefore, we identified which components of Hachimi-jio-gan are responsible for pharmacological effects such as hormonal ligands for nuclear receptors. 


\section{Materials and Methods}

\section{Cell lines}

PC-3 (prostate), HepG2 (liver), and HTB185 (cerebellum) cells were obtained from the American Type Culture Collection (Manassas, VA, USA), COS7 (kidney), and Nthy-ori3-1 (thyroid) was obtained from Dainippon-Sumitomo grown in DMEM, or RPMI1640 supplemented with $10 \%$ fetal bovine serum, $0.25 \mathrm{mg} /$ $\mathrm{ml}$ streptomycin (GIBCO BRL), and $100 \mathrm{mg} / \mathrm{ml}$ penicillin. CV1 cells (kidney) have been described previously (12). Cells were grown on $150 \mathrm{~cm}^{2}$ cell culture plates for 48 hours, and then cells were cultured in DMEM containing 10\% (v/v) charcoal-stripped FBS in the presence or absence of appropriate ligands. To remove steroid and thyroid hormones, FBS was treated for 24 hours at $4{ }^{\circ} \mathrm{C}$ with $50 \mathrm{mg} / \mathrm{ml}$ of activated charcoal (Sigma) and $30 \mathrm{mg} / \mathrm{ml}$ of anion exchange resin (type AGX-8, analytical grade, BioRad).

\section{Transfection}

The transfection assays and transfected plasmids were described previously [12]. Human PPAR $\alpha$ cDNA was amplified by polymerase chain reaction (PCR) from human liver cDNA. The PCR product was verified as PPAR $\alpha$ cDNA by sequencing, and was cloned into the expression vector $\mathrm{pKCR} 2$. The PPAR or RXR response elements reporter construct consist two copies of the direct repeat motif of hexamer half sites, TGACCT, spaced by one nucleotide (DR1 element) upstream of the TK109 promoter in the vector pA3Luc (DR1-TKLuc). The positive TRE (thyroid hormone response elements) constructs consist of two copies of a palindromic element upstream of TK109 promoter in the pA3Luc (pal-TKLuc). MMTV-Luc contains the murine mammary tumor virus promoter in pA3Luc. The ERE (estrogen response element)-Luc contains two copies of an ER response element upstream of TK109 (ERE-TKLuc). Cells were transfected with the following amounts of DNA per 6 wells ( $10 \mu \mathrm{g}$ of pA3 Luc-TK reporter constructs, $100 \mathrm{ng}$ of each receptor expression plasmid). Sixteen hours after transfection, cells were cultured in the absence or presence of the appropriate concentration of Hachimi-jiogan and ligands for 24 hours and then harvested for luciferase assays.

Dulbecco's modified Eagle's medium (DMEM) and sera were purchased from Gibco BRL (Grand Island, NY, USA). Hachimi-jio-gan extract and its component powder were kindly supplied by Tsumura Inc. (Tokyo, Japan). Fenofibrate was kindly supplied by Kaken Inc. (Tokyo, Japan). Before using these herbal components in transfection experiments, those components was added to water, boiled for one hour and filtered.

\section{Results}

\section{Hachimi-jio-gan activated PPAR $\alpha$-related transactivation in CV1 cells}

Fig. 1 shows the luciferase activities of Hachimi-jiogan on nuclear receptor in transfected CV1 cells. When $0.5 \mathrm{mg} / \mathrm{ml}$ Hachimi-jio-gan was added, luciferase activity increased to 3.1 -fold in CV1 cells transfected with the PPAR $\alpha$ expression vector and DR1 response element linked to the TK promoter construct; however, Hachimi-jio-gan had no effects on

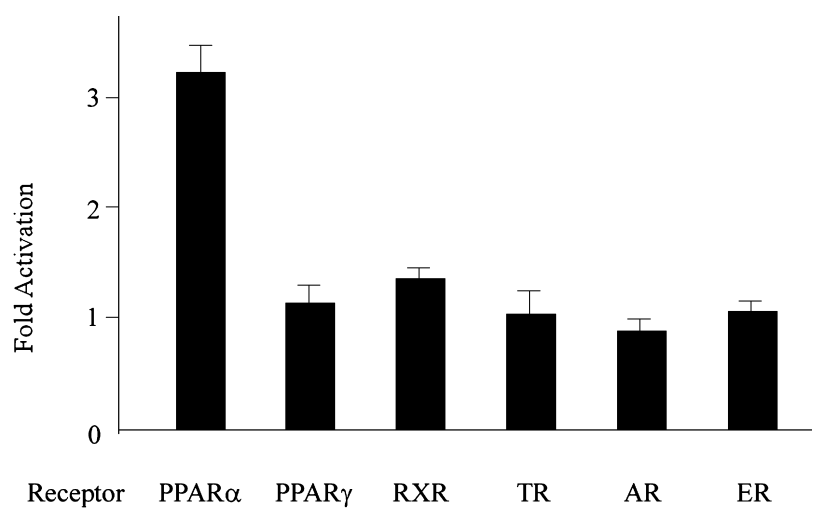

Fig. 1. Adding $0.5 \mathrm{mg} / \mathrm{ml}$ of Hachimi-jio-gan specifically activated PPAR $\alpha$-related transactivation but did not alter PPAR $\gamma, \mathrm{TR}$ (thyroid hormone receptor), AR (androgen receptor), ER (estrogen receptor), and RXR-related transactivation. CV1 cells were transfected as described under "Materials and Methods". Ten $\mu \mathrm{g}$ of pA3 Luc-TK reporter constructs and $100 \mathrm{ng}$ of respective nuclear receptor expression plasmids (DR1-TKLuc and PPAR $\alpha$, DR1-TKLuc and PPAR $\gamma$, DR1-TKLuc and RXR $\alpha$, pal-TKLuc and TR $\beta 1$, MMTV-Luc and AR, ERE-TKLuc and ER) were cotransfected in the presence or absence of $0.5 \mathrm{mg} / \mathrm{ml}$ Hachimi-jio-gan. The data were quantified as fold luciferase activity, where 1 equals the activity of the reporter in the absence of Hachimi-jio-gan. The transfected receptor expression vectors are indicated below. Results represent the mean \pm S.E.M. of three independent experiments. 
A
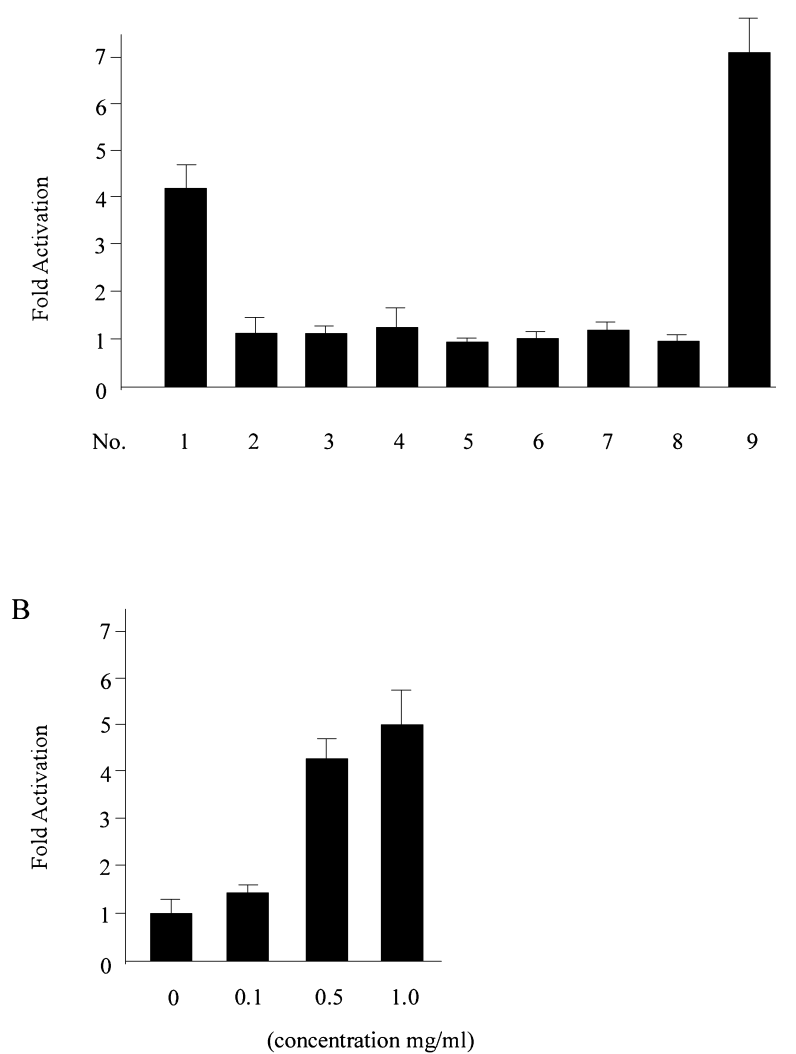

Fig. 2. (A) Eight gradients of Hachimi-jio-gan were tested in the transfection assays into CV1 cells. CV1 cells were transfected with a DR1-TKLuc reporter and $0.1 \mu \mathrm{g}$ of $\mathrm{hPPAR} \alpha$ expression vector in the presence or absence of 8 gradients of Hachimi-jio-gan. Only cinnamomi cortex activated PPAR $\alpha$-mediated transactivation specifically. The number indicated below shows the gradients of Hachimi-jio-gan (1: cinamomi cortex, 2: rehmanniae radix, 3: orni fructus, 4: dioscoreae rhizoma, 5: alismatis rhizoma, 6: hoelen, 7: moutan cortex, 8: aconiti, 9: fenofibrate). (B) Activation by cinnamomi cortex on PPAR $\alpha$ was dose-dependent. Results represent the mean \pm S.E.M. of three independent experiments. Data were quantified as fold luciferase activity.

thyroid hormone receptor, androgen receptor, estrogen receptor, RXR, and PPAR $\alpha$. These findings suggested that Hachimi-jio-gan might stimulate PPAR $\alpha$ specifically.

One component of Hachimi-jio-gan, cinnamomi cortex, had a PPARa ligand-like function

Next, to examine which component of Hachimi-jiogan is responsible for the activation of PPAR $\alpha$, eight

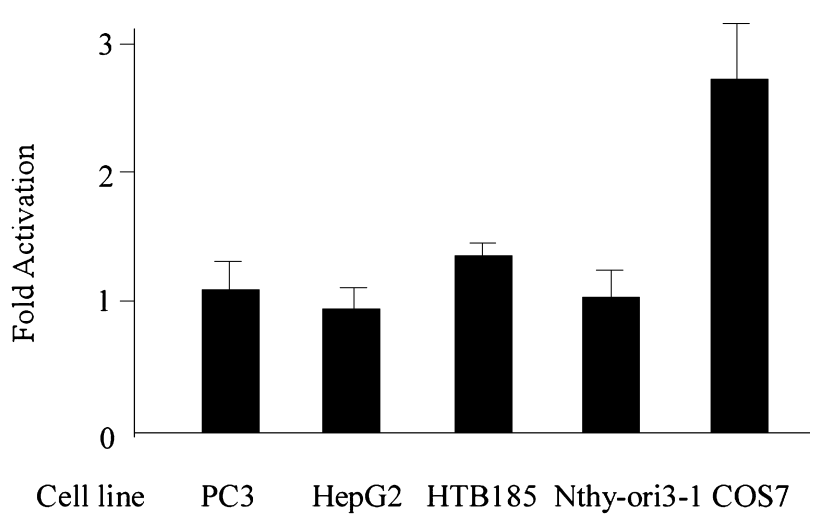

Fig. 3. The activating effects of cinnamomi cortex on PPAR $\alpha$ were observed only in renal cells (COS7). DR1 TKLuc reporter and $0.1 \mu \mathrm{g}$ of hPPAR $\alpha$ expression vector were transfected into five different cell lines (PC3 (prostate), HepG2 (liver), HTB185 (cerebellum), Nthy-ori3-1 (thyroid), COS7 (kidney)) in the presence or absence of $0.1 \mathrm{mg} / \mathrm{ml}$ of cinnamomi cortex. Results represent the mean \pm S.E.M. of three independent experiments. Data were quantified as fold luciferase activity.

gradients of Hachimi-jio-gan were tested in the same system using CV1 cells. As a control, $1 \mu \mathrm{M}$ fenofibrate caused 7.1-fold activation of TK promoter activity fused to the DR1 element in this system. While $1.0 \mathrm{mg} / \mathrm{ml}$ cinnamomi cortex enhanced PPAR $\alpha$-mediated activation by 4.1 -fold, the other seven gradients did not show any increase of PPAR $\alpha$-mediated transactivation (Fig. 2A). Moreover, stimulation of cinnamomi cortex on PPAR $\alpha$ was observed in a dosedependent manner (Fig. 2B). These observations demonstrated that cinnamomi cortex, one gradient of Hachimi-jio-gan, functions with a PPAR- $\alpha$ ligand-like effect.

\section{Effects of cinnamomi cortex on PPARa were observed specifically in renal cells}

To determine whether cinnamomi cortex activates PPAR $\alpha$ in any cells derived from many kinds of organs, activity was measured in five different cell lines (PC3 (prostate), HepG2 (liver), HTB185 (cerebellum), Nthy-ori3-1 (thyroid), COS7 (kidney)). Cinnamomi cortex activated the PPAR $\alpha$-mediated transcription in COS7 cells (2.8-fold) as well as CV1 cells and had no effect in other cells (Fig. 3). These findings suggested that PPAR $\alpha$-mediated effects by cinnamomi cortex might be renal-specific. 


\section{Discussion}

The present study demonstrated that Hachimi-jiogan activated PPAR $\alpha$ induced transactivation. Our study of transfection into CV1 cells clearly showed that Hachimi-jio-gan activated DR1 luciferase activity and this activation is PPAR $\alpha$-specific. Hachimi-jio-gan was reported to have favorable effects on lipid [11] and glucose metabolism [5, 13]; however, this mechanism has not been resolved. Our study suggested one possibility that the effect of Hachimi-jio-gan was induced by PPAR $\alpha$ activation.

Moreover, we established that cinnamomi cortex, one component of Hachimi-jio-gan, was mainly responsible for PPAR $\alpha$ activation and this activation was dose-dependent with renal tissue-specific activation. Cinnamomi cortex is commonly obtained not only from traditional herbal medicine for treating blood circulation disturbance (14) and inflammatory disease [15], but also from natural food; however, the mechanisms underlying these effects of cinnamomi cortex have yet to be clarified. Only one report revealed that cinnamomi cortex inhibits NF- $\kappa$ B activation and iNOS protein expression in stress-induced $\beta$-cells by using a molecular experiment method [16]. Thus, the molecular mechanisms of cinnamomi cortex effects will eventually be elucidated.

This is the first report that cinnamomi cortex stimulated PPAR $\alpha$-mediated transactivation. To clarify this mechanism, we performed a molecular experiment. In preliminary data, nuclear receptor coactivator SRC-1 [17] binding to PPAR $\alpha$ was not enhanced by adding cinnamomi cortex in a GST pull-down assay. These findings suggested that activating PPAR $\alpha$ by cinnamomi cortex would not be mediated through protein-protein interaction between PPAR $\alpha$ and its coactivators.
In our preliminary data, other herbal medicines such as Shoseiryuto (2.3-fold) and Keishikajutubuto (2.1fold), including cinnamomi cortex as components, also activated PPAR $\alpha$-mediated transactivation in CV1 cells under the same conditions. As herbal medicine is not simply a purified substrate but contains many ingredients, the activating degree may be different in each.

Hachimi-jio-gan was reported to prevent diabetic kidney damage by reducing renal oxidative stress or attenuating glucose toxicity [18]. In addition, PPAR $\alpha$ is highly expressed in the kidney [19]; however, the function of PPAR $\alpha$ in the kidney has not been identified. Recently, the role of PPAR $\alpha$ in the kidney has been analyzed in several experiments. PPAR $\alpha$-deficient mice showed severe diabetic nephropathy through an increase in extracellular matrix formation and inflammation [20]. Moreover, a PPAR $\alpha$ agonist, fenofibrate improved glomerular hypertrophy and mesangial matrix expansion, resulting in reduced urinary albumin excretion in $\mathrm{db} / \mathrm{db}$ mice [21]. Combining these data with our findings, one possibility is that improvement by Hachimi-jio-gan of renal disease may be mediated via PPAR $\alpha$ stimulation. Although the reason why PPAR $\alpha$-mediated transactivation was specifically activated by cinnamomi cortex in renal cells is not fully explained at present, our findings may lead to the development of novel therapy for the prevention of diabetic nephropathy through PPAR $\alpha$ activation, not only by Hachimi-jio-gan but also by cinnamomi cortex.

\section{Acknowledgement}

This study is partly supported by the research fund of the Institute of Kampo Medicine, Japan.

\section{References}

1. Yokozawa T, Yamabe N, Cho EJ, Nakagawa T, Oowada S (2003) A study on the effects to diabetic nephropathy of Hachimi-jio-gan in rats. Nephron Exp Nephrol 97: e38-e48.

2. Yamabe N, Kang KS, Goto E, Tanaka T, Yokozawa T (2007) Beneficial effect of corni fructus, a constituent of Hachimi-jio-gan, on advanced glycation end-productmediated renal injury in streptozotocin-treated diabetic rats. Biol Pharm Bull 30: 520-526.

3. Yamabe N, Yokozawa T (2006) Activity of Chinese prescription Hachimi-jio-gan against renal damage in the Otsuka Long-Evans Tokushima fatty rat: a model of human type 2 diabetes mellitus. J Pharm Pharmacol 58: 535-545.

4. Nakagawa T, Yokozawa T, Yamabe N, Rhyn DY, Goto H, Shimada Y, Shibahara N (2005) Long-term treatment with Hachimi-jio-gan attenuates kidney damage in spontaneously diabetic WBN/Kob rats. J Pharm Pharmacol 57: 1205-1212.

5. Hirotani Y, Ikeda T, Yamamoto K, Kurokawa N 
(2007) Effects of Hachimi-jio-gan (Ba-Wei-HuangWan) on hyperglycemia in streptozotocin-induced diabetic rats. Bio Pharm Bull 30: 1015-1020.

6. Yamabe N, Yokozawa T, Kim HY, Cho EL (2005) Protective effect of Hachimi-jio-gan against renal failure in a subtotal nephrectomy rat model. J Pharm Pharmacol 57: 1637-1644.

7. Hirokawa S, Nose M, Amagaya S, Oyama T, Ogihara Y (1993) Protective effect of hachimi-jio-gan, an oriental herbal medicinal mixture, against cerebral anoxia. $J$ Ethnopharmacol 40: 201-206.

8. Yamada K, Yagi G, Kanba S (2005) Effectiveness of herbal medicine (Rokumigan and Hachimijiogan) for fatigue or loss of energy in patients with partial remitted major depressive disorder. Psychiatry and Clinical Neurosciences 59: 610-612.

9. Iwasaki K, Kobayashi S, Chimura Y, Taguchi M, Inose K, Cho S, Akiba T, Arai H, Cyong J-C, Sasaki H (2004) A randomized, double-blind, placebo-controlled clinical trial of the Chinese herbal medicine "ba wei di huang wan" in the treatment of dementia. JAGS 52: 1518-1521.

10. Otani S, Usuki S, Iwasaki H, Inoue S, Yamashita K (1991) Successful treatment of a hyperprolactinemic infertile woman with a pituitary microadenoma using hachimijiogan. Am J Chin Med 19: 145-154.

11. Yoshida H, Kusukawa R, Watanabe N, Ohtsuki G, Sakamoto T, Haranaka R (1985) The effects of Ba-weiwan (hachimijiogan) on plasma levels of high density lipoprotein-cholesterol and lipoperoxide in aged individuals. Am J Chin Med 13: 71-76.

12. Monden T, Nakajima Y, Hashida T, Ishii S, Tomaru T, Shibusawa N, Hashimoto K, Satoh T, Yamada M, Mori M, Kasai K (2006) Expression of thyroid hormone receptor isoforms down-regulated by thyroid hormone in human medulloblastoma cells. Endocr J 53: 181-187.

13. Cheng JT, Liu IM, Chi TC, Su HC, Chang CG (2001) metoformin-like effects of Quei Fu Huang Wan, a Chi- nese herbal mixture, on streptozotocin-induced diabetic rat. Horm Metab Res 33: 727-732.

14. Matsuda H, Matsuda R, Fukuda S, Shiomoto H, Kubo M. (1987) Anti-thrombic actions of $70 \%$ of methanolic extract and cinnamic aldehyde from cinnamomi cortex. Chem Pharm Bull (Tokyo) 35: 1275-1280.

15. Kubo M, Ma S, Wu J, Matsuda H (1996) Anti-inflammatory activities of $70 \%$ methanolic extract from Cinnamomi Cortex. Biol Pharm Bull 19: 1041-1045.

16. Kwon KB, Kim EK, Jeong ES, Lee YH, Lee YR, Park JW, Ryu DG, Park BH (2006) Cortex cinnamomi extract prevents streptozotocin- and cytokine-induced $\beta$-cell damage by inhibiting NF- $\kappa \mathrm{B}$. World J Gastroenterol 12: 4331-4337.

17. Tien ES, Hannon DB, Thompson JT, Vanden Heuvel JP (2006) Examination of ligand-dependent coactivator recruitment by peroxisome proliferators-activated receptor- $\alpha$ (PPAR $\alpha)$. PPAR Research: 1-9.

18. Kim HY, Yokozawa T, Cho EJ, Yamabe N (2004) Protective effects of the Chinese prescription Hachimijio-gan against diabetic oxidative stress. J Pharm Pharmacol 56: 1299-1305.

19. Tachibana K, Anzai N, Ueda C, Katayama T, Kirino T, Takahashi R, Yamasaki D, Ishimoto K, Tanaka T, Hamakubo T, Ueda Y, Arai H, Sakai J, Kodama T, Doi $\mathrm{T}$ (2006) Analysis of PPAR alpha function inhuman kidney cell using siRNA. Nucleic Acids Symposium Series 50: 257-258.

20. Park CW, Kim HW, Ko SH, Chung HW, Lim SW, Yang CW, Chang YS, Sugawara A, Guan YF, Breyer MD (2006) Accelerated diabetic nephropathy in mice lacking the peroxisome proliferators-activated receptor a. Diabetes 55: 885-893.

21. Park CW, Zhang Y, Zhang X, Wu J, Chen L, Cha DR, Su D, Hwang M-T, Fan X, Davis L, Striker G, Zheng F, Breyer M, Guan Y (2006) PPAR $\alpha$ agonist fenofibrate improves diabetic nephropathy in $d b / d b$ mice. Kidney Int 69: 1511-1517. 